\title{
Electrocutaneous stimulation I. The effects of stimulus parameters on absolute threshold
}

\author{
JOHN P. GIRVIN \\ The University of Western Ontario, London, Ontario, Canada \\ LAWRENCE E. MARKS \\ John B. Plence Foundation Laboratory and Yale University, New Haven, Connecticut \\ and \\ JOHN L. ANTUNES, DONALD O. QUEST, MICHAEL D. O'KEEFE, \\ PETER NING, and Wm. H. DOBELLE \\ College of Physicians and Surgeons of Columbia University, New York, New York
}

\begin{abstract}
Thresholds for detecting electrical stimulation wore measured as a function of (1) body locus, (2) electrode configuration, (8) stimulus waveform, and (4) pulse duration. The results were: (1) the forehead gave slightly, but not reliably, lower threaholda than the abdomen; (2) concentric electrodes gave slightiy, but not reliably, lower thresholds than unifocal electrodes; (8) cathodal monophasic $(-)$ pulses and biphasic pulses $(+1-$ and $-1+)$ gave identical thresholds, while anodal monophasic $(t)$ pulses gave higher thresholds; and (4) thresholds decreased as pulse duration increased up to $.5 \mathrm{msec}$, but changed less with longer pulses.
\end{abstract}

This is the first of three papers dealing with sensory responses to electrocutaneous stimulation. The present paper examines several of the stimulus parameters that influence absolute sensitivity; the second paper (Marks, Girvin, Quest, Antunes, Ning, O'Keefe, \& Dobelle, 1982) explores the skin's capacity to process spatial information, and the third (Marks, Girvin, O'Keefe, Ning, Quest, Antunes, \& Dobelle, 1982) deals with the skin's ability to make spatiotemporal discriminations.

One long-range goal of this research is the development of an aid for the deaf to translate speech information into a spatiotemporal pattern of electrocutaneous stimulation. However, the shorter-range goals of these studies are (1) to assess some of the probable perceptual limitations on processing electrical stimulation, and (2) to determine some of the properties of an electrical stimulation system that are likely to maximize the ability to process information presented through the skin regardless of the specific application.

Considerable work has already gone into the study of absolute sensitivity to electrocutaneous stimulation. Although there were no details provided about the exact nature of the "vibratory" thresholds measured, Hahn (1958) showed the marked effect of the pulse duration

This research was supported by a grant from the National Science Foundation (NSF PFR 79-17634). Reprints may be requested from Lawrence E. Marks, John B. Pierce Foundation Laboratory, 290 Congress Avenue, New Haven, Connecticut 06519. J. L. Antunes and D. O. Quest are affilinted with the Neurological Institute of New York, and M. D. O'Keefe, P. Ning, and W. H. Dobelle, with the Division of Artificial Organs, both of the College of Physicians and Surgeons of Columbia University. of anodal pulses on such thresholds. With 7-mm-diam metal electrodes applied to the index fingers of his subjects, the most striking reduction in threshold occurred as the pulse duration increased from .1 to $.7 \mathrm{msec}$; there was negligible change with durations above 2 msec.

Gilmer (1961), studying primarily the dynamic range (between perception and pain) of pulses delivered through 6-9-mm spherical electrodes, found that durations of approximately $10 \mathrm{msec}$ required less current to reach threshold than did shorter, or longer, durations. In a similar study, Brown, Spern, Schmitt, and Solomon (1966), using an 18-mm spherical electrode, found thresholds to be somewhat lower with 1.0 than with $.5-\mathrm{msec}$ pulses, with trains of pulses of up to 8 in number, and with the arm, chest, or abdomen, rather than the back. Gibson (1968) reported that cathodal (monophasic) pulses required threshold currents only about $50 \%-75 \%$ of those of equivalent anodal pulses. Nevertheless, to reduce skin irritation, he used anodal pulses delivered through a 10-mm-diam spherical electrode and showed that thresholds were higher on hairy than on hairless skin and that, with $100-\mathrm{Hz}$ stimulus trains, the threshold was a decreasing function of the number of pulses.

Saunders and Collins (1971) found that the threshold on the skin of the abdomen in a given individual was represented by a constant charge, varying across subjects from 29 to $130 \mathrm{nC}$ (average 62). Rollman (1974), using two Grass EEG electrodes $2 \mathrm{~cm}$ apart, showed the remarkably steep psychometric functions for detection of electrocutaneous pulses, particularly with pulse durations greater than $.2 \mathrm{msec}$. 
It is clear that stimulus parameters such as duration and waveform influence sensitivity. Perhaps these parameters interact. The goal of the present study was to examine systematically the effects on sensitivity of several stimulus parameters. The study asks how each of the following influences the absolute threshold of electrical stimulation: (1) body site stimulated (forehead vs. abdomen); (2) configuration of the electrode (small, unifocal electrode vs. large, concentric electrode); (3) stimulus waveform (monophasic positive, monophasic negative, and biphasic pulses); and (4) pulse duration of the stimulus.

\section{METHOD}

\section{Subjects}

The subjects were all adult males. The observations reported here were carried out on three of the authors. However, pilot observations were also made on a number of other persons associated with the study.

\section{Stimulation}

The stimuli were constant-current square-wave pulses whose duration was varied from .0625 to $1.0 \mathrm{msec}$. The waveforms were monophasic positive $(+)$, monophasic negative $(-)$, and symmetrically biphasic $(+/-$ or $-/+)$. The constant-current source was the computer-controlled output of a battery $( \pm 45 \mathrm{~V})$ stimulator that was capacitively and optically isolated from the subject (Mladejovsky, Eddington, Evans, \& Dobelle, 1976).

Several stimulating electrode arrays were employed. Preliminary experiments were performed using an $8 \times 8$ array of hexagonally packed, $1-\mathrm{mm}^{2}$ platinum disks on $5-\mathrm{mm}$ centers. For the experiments reported, similar electrode contacts were arranged in linear arrays of 16 electrodes. These arrays were used for unifocal stimulation, the return path being an indifferent electrode on the left leg. This method of stimulation duplicates experiments conducted earlier on the visual cortex of unsighted human patients (Dobelle, Mladejovsky, Evans, Roberts, \& Girvin, 1976 ; Dobelle, Mladejovsky, \& Girvin, 1974). The present experiments were performed using these unifocal electrodes and larger, concentric electrodes kindly supplied to us by Frank Saunders (see Saunders, 1974, 1977; Szeto \& Saunders, 1982). These electrodes have rounded corners, constructed of either sterling silver or gold. Each has an active central area of approximately $10-12 \mathrm{~mm}^{2}$, separated from the surrounding return pad (approximately 10 times the area of the center) by $1 \mathrm{~mm}$ of insulation. Either four or five electrodes were utilized in an array at a given time, and all of the surrounds were joined commonly by connecting leads to one side of the stimulator.

The electrodes were placed on the forehead and the abdomen. Electrical contact was facilitated by moistening the skin with either a small amount of conducting gel (Cor-gel ${ }^{\circledR}$ ) (unifocal electrodes) or water (concentric electrodes). Electrodes were held on the skin by strips of paper tape and a loosely applied elastic bandage."

\section{Procedure}

The subject was seated in a comfortable reclining chair located in a sound-attenuated booth. Stimulation was controlled by the observer through the use of a touchtone keyboard. The output of the keyboard served two purposes. First, it encoded the subject's responses to the stimulation for transmission to the computer memory. Second, it allowed the subject to request the next stimulation.

Absolute thresholds were measured using a modified staircase procedure similar to PEST (Taylor \& Creelman, 1967). The first stimulus had a current of $1 \mathrm{~mA}$. If it was detected, the subject indicated "yes" to the computer through the touchtone and on the next trial the current was halved. If the stimulus was not detected, a "no" was provided to the computer and on the next trial the level was doubled. Once the lower limit was determined by the first "no" and the upper limit by the first subsequent "yes," each succeeding stimulus was presented at a level of current halfway between that of a previous stimulus and the limit being approached; each reversal then set a new limit. Thus, the stimuli bracketed threshold in smaller and smaller steps, and the run terminated when the change in stimulus current was $10 \%$ of the previous stimulus. This value was defined as threshold. The only limiting factor was the maximal output of the computer stimulator, which was $8 \mathrm{~mA}$.

The four waveforms $(+,-,+/-,-/+)$, five pulse durations $(.0625, .125, .25, .5,1.0 \mathrm{msec})$, and two body sites (forehead and abdomen) were utilized in all possible combinations. These combinations were scrambled and delivered randomly by the computer. Three thresholds at each combination were measured, giving a total of 120 threshold determinations ( 5 pulse durations $\times 4$ waveforms $\times 2$ body sites $\times 3$ measurements $=120$ ). (The single exception to this procedure was one subject in whom six measurements were made on the abdomen using the concentric electrodes.) Each subject made these determinations with both the unifocal and concentric electrodes.

\section{RESULTS}

\section{Body Site and Electrode Configuration}

The average threshold determined on the forehead (2.97 mA) was slightly, but not significantly, smaller than that determined on the abdomen $(3.28 \mathrm{~mA})$ $[F(1,2)<1]$. Each of these values pools all of the data over pulse duration and electrode configuration. There is no interaction either between body site and pulse duration, or between body site and electrode configuration $[F(1,2)<1]$. In sum, the forehead and abdomen are roughly equal in sensitivity at all durations and with both small unifocal and large concentric electrodes. The two kinds of electrode themselves also gave comparable results, the average threshold being $3.24 \mathrm{~mA}$ for the small unifocal electrode and $3.05 \mathrm{~mA}$ for the large concentric electrode; the difference is not reliable $[\mathrm{F}(1,2)<1]$.

\section{Pulse Duration}

Figure 1 shows the relationship between decreasing threshold and increasing pulse duration. The major drop in threshold occurs when pulse duration increases from

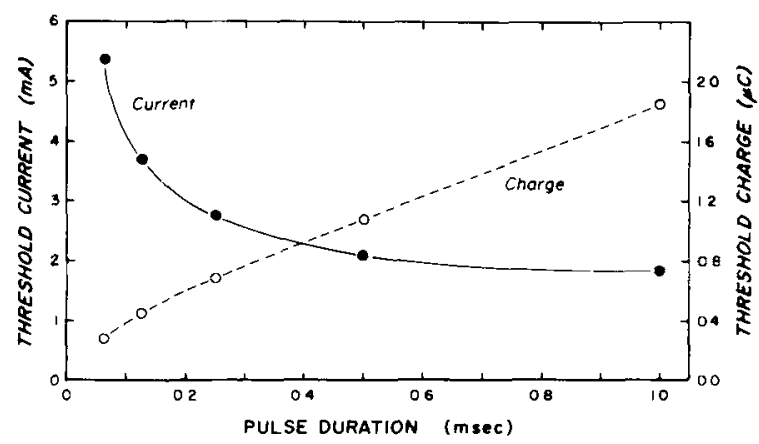

Figure 1. Absolute threshold current (filled circles) in milliamperes and threshold charge (open circles) in microcoulombs as a function of pulse duration, averaged over all subjects and conditions. 
.0625 up to about $.5 \mathrm{msec}$. However, there is little further absolute reduction in threshold with increase in the pulse duration beyond this point-although, in percentage terms, threshold does continue to drop (Figure 2). This outcome agrees with results of Rollman (1974).

The dashed line in Figure 1 rearranges the same data in order to represent charge (the product of pulse duration and threshold current) vs. pulse duration. With increasing pulse duration, the charge must increase nearly linearly to attain threshold.

\section{Stimulus Waveform}

Purely on physiological grounds, there are theoretical difficulties in equating the true duration of a biphasic, constant current pulse with that of a monophasic pulse. For example, should both the anodal and cathodal phases of a biphasic pulse be considered as forming the physiological duration of the stimulating pulse? If so, then obviously the monophasic equivalent would be that in which the total duration equaled the combined duration of the two phases. If, on the other hand, only a single phase of the biphasic pulse is important physiologically, then the equivalent monophasic pulse would be simply the duration of one phase.

Figure 2 plots, on logarithmic axes, average thresholds as a function of pulse duration for each of the four waveforms. This presentation treats the duration of the monophasic pulses as equivalent to the duration of each phase of the biphasic pulses (e.g., a .5-msec monophasic pulse and a $.5 \times .5 \mathrm{msec}$ biphasic pulse are both $.5-\mathrm{msec}$ pulses). By treating the data in this way, the results for the two biphasic waveforms $(+/-$ and $-/+)$ and for the monophasic cathodal $(-)$ waveform collapse roughly into a single function, while the results for the monophasic anodal ( + ) waveform are considerably higher at all durations $[F(3,6)=75.9, p<.001]$. Statistical analysis also revealed an interaction between waveform and body site, accounted for largely by much higher thresholds with anodal pulses to the abdomen than to the forehead $[F(3,6)=8.80, p<.02]$.

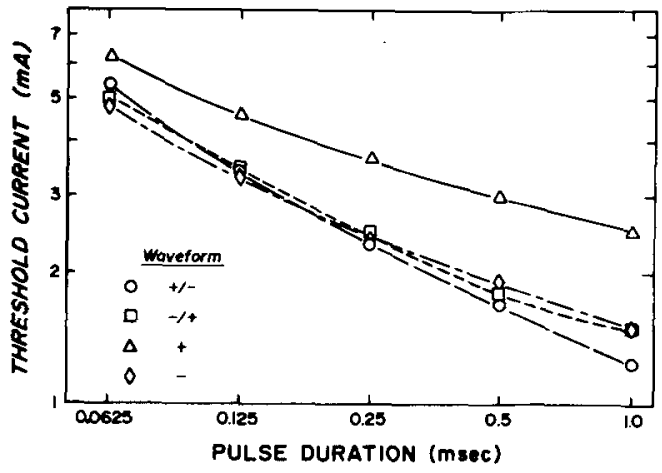

Figure 2. Absolute threshold current in milliamperes as a function of pulse duration for each of four waveforms, averaged over subjects and conditions.
It is also possible to treat the data in a different way, namely by considering the "true" duration of the biphasic pulses as equivalent to the sum of the durations of the two phases rather than as the duration of a single phase (e.g., a .5-msec monophasic pulse and a $.25 \times$ .25 msec biphasic pulse are both .5 -msec pulses). When treated in this way, the data for both biphasic waveforms in Figure 2 would shift to the right by a factor of two, and thus no longer converge with the data for the monophasic cathodal waveform; nor would the data for the biphasic waveforms converge with the data for the monophasic anodal waveform, because the functions relating log threshold to $\log$ pulse duration have different forms $[F(12,24)=12.84, p<.001]$. Although it is difficult to formulate a theoretical basis for deciding how to compute the duration of the biphasic pulses, the similarity among three of the functions, as depicted in Figure 2, suggests the heuristic value of treating the duration of a monophasic (cathodal) pulse as equivalent to the duration of one phase of the symmetric, biphasic pulses.

Another salient feature of Figure 2 is the demonstration that some temporal summation continues, albeit less and less extensively, even with durations of $1 \mathrm{msec}$. This result is in keeping with Rollman's (1974) finding of complete reciprocity only with durations up to a fraction of a millisecond, but some summation up to at least $5 \mathrm{msec}$.

\section{DISCUSSION}

\section{Body Site and Electrode Configuration}

We selected the forehead and abdomen for study. The abdomen was chosen because so much literature has been devoted to considering this body site for both vibratory and electrocutaneous sensory substitution devices. The forehead was chosen because our preliminary observations on various body locations indicated that stimuli applied to the skin of the forehead created more discrete sensations and might require less current. Although there are cosmetic drawbacks to using the forehead, these could be overcome. Other advantages of the forehead would include the easy attention to stimulation, a shorter latency, and the fact that this area is unencumbered by overlying clothes or by participation in body movements. The forearm was not chosen as a site for investigation, since stimuli applied to a moving forearm cannot be easily attended; immobilization of the forearm would simply add another disability.

The thresholds are slightly, but not reliably, lower on the forehead. However, all subjects found that the stimuli were much more easily attended on the forehead than on the abdomen. This would surely be even more strikingly advantageous in the moving, clothed individual in a normal environment as opposed to the quiet, relatively stimulus-free environment of a sound-attenuated booth. 
Pulse Duration

As pulse duration increases, there is clearly a reduction in the peak curr : intensity required to reach threshold, even though \& eater charge is required. Our results suggest that pulses on the order of $.5 \mathrm{msec}$ duration ( $1 \mathrm{msec}$ total duration in the case of biphasic pulses) would represent a reasonaole compromise between the high charge/pulse needed at longer durations and the high current intensities needed at shorter durations. In this regard, it is interesting that Gibson (1968) arrived at the same conclusion on the basis of looking at the dynamic range, that is, a compromise between painful pulses, on the one hand, and touch thresholds, on the other. Using an electrode much larger than $1 \mathrm{~mm}$, similar to Gibson's, Rollman (1974) also found the major drop in threshold to occur as pulse duration increased up to about $.5 \mathrm{msec}$.

\section{Stimulus Waveform}

Clearly, with respect to the current needed to reach threshold, there is no difference between the use of biphasic waveforms, either $+/-$ or $-1+$, and cathodal pulses of the duration of a single phase of the biphasic pulse. That anodal pulses yield higher thresholds would lead one to conclude that it is the cathodal phase of the biphasic pulse, irrespective of whether it is the lead or lag phase, that provides the physiological stimulation. The overall difference between thresholds of anodal and cathodal pulses ( 4.14 vs. $2.91 \mathrm{~mA}$ ) is in keeping with the observations of Gibson (1968).

Although there has been no systematic study of parameters of stimulation utilizing different waveforms, Gibson (1968) did note that there was "less reddening" and discomfort with "bidirectional" pulses than with monophasic pulses. This would, of course, fit with the notion that biphasic waveforms lead to less tissue damage due to $\mathrm{pH}$ changes resulting from electrolysis at the tissue/electrode junction, and electrophoresis of tissue components.

\section{Do the Present Results Place Any Serious}

Restrictions on Stimulus Parameters

in a Sensory Substitution Device?

Although thresholds are slightly lower on the forehead, the difference is not reliable and certainly is not so great as to preclude the abdomen as the locus for electrocutaneous stimulation. Results from various pulse durations and stimulus waveforms clearly allow for flexibility in design. Of course, the qualitative aspects of the sensations and the spatiotemporal discriminability may impose greater restrictions on pulse duration, stimulus waveform, and body locus. The following papers in the series treat this issue.

\section{REFERENCES}

Brown, R. L., Spenn, R. A., Schmit, K., \& Solomon, A. Stimulus parameter considerations and individual differences in cutaneous sensitivity to electropulse stimulations. Perceptual and Motor Sklls, 1966, 23, 1215-1222.

Dobelle, W. H., Mladejovgxy, M. G., Evang, J. R., Robente, T. S., \& Ginvin, J. P. 'Braille' reading by a blind volunteer by visual cortex stimulation. Nature, 1976, 250, 111-112.

Dobelle, W. H., Mladejovaxy, M. G., \& Ginvin, J. P. Artificial vision for the blind: Electrical stimulation of visual cortex offers hope for a functional prosthesis. Science, 1974, $183,40-444$.

Gibson, R. H. Electrical stimulation of pain and touch. In D. R. Kenshalo (Ed.), The skin senses. Springfield, Ill: Thomas, 1968.

Gilmen, B. von H. Toward cutaneous electropulse communications. Journal of Psychology, 1961, 52, 211-222.

Hahn, J. F. Cutaneous vibratory thresholds for square-wave electrical pulses. Science, 1958, 127, 879-880.

Mark8, L. E., Ginvin, J. P., O'Keefe, M. D., Ning, P., Quest, D. O., Antunes, J. L., \& Dobelle, W. H. Electrocutaneous stimulation III. The perception of temporal order. Perception \& Psychophysics, 1982, 32, 537-541.

Marks, L. E., Girvin, J. P., Quest, D. O., Antunes, J. L., Ning, P., O'Keefe, M. D., \& Dobelle, W. H. Electrocutaneous stimulation II. The estimation of distance between two points. Perception \& Psychophysics, 1982, 32, 529-536.

Mladejoviky, M. G., Edoington, D. K., Evans, J. R., \& Dobelle, W. H. A computer-based brain stimulation syatem to investigate sensory prostheses for the blind and deaf. $I E E E$ Transactions on Blomedical Engineering, 1976, BME-23, 286-296.

Rollyan, G. D. Electrocutaneous stimulation. In F. A. Geldard (Ed.), Cutaneous communication systems and devices. Austin, Tex: The Psychonomic Society, 1974.

Saunders, F. A. Electrocutaneous displays. In F. A. Oeldard (Ed.), Cutaneous communication systems and devices. Austin, Tex: The Psychonomic Society, 1974.

SAunders, F. A. Recommended procedures for electrocutaneous displays. In F. T. Hambrecht J. Reswick (Eds.), Functional electrical stimulation. New York: Dekker, 1977.

Saunders, F. A., \& Collins, C. C. Electrical stimulation of the sense of touch. Journal of Blomedical Systems, 1971, 2, 27-37.

Szero, A., SAundere, F. A. Electrocutaneous stimulation of sensory communication and rehabilitation engineering. IEEE Transactions on Biomedical Engineering, 1982, BME-23, 300-308.

Taylor, M. M., \& Creelman, C. D. PEST: Efficient estimates on probability functions. Journal of the Acoustical Society of America, 1967, 41, 782-787.

\section{NOTE}

1. The literature on electrical stimulation of the skin is replete with accounts of fluctuations in threshold and impedance, suggesting problems with electrode contact. Also, high skin impedances $(20-40 \mathrm{k} \Omega)$ mean that voltages in excess of $100 \mathrm{~V}$ are often needed to reach threshold. Such voltages would be difficult to achieve with lightweight, battery-powered equipment, and probably would also damage the skin.

Consequently, we began exploring a variety of commercially available electrode pastes, only to realize that a majority of these materials have been designed for ECG/EEG recording, rather than stimulation. Their efficacy in reducing impedance was variable and changed with time. Furthermore, many contained ground glass to abrade the stratum corneum and were thus not suitable for chronic use.

Formulation of commercial electrode paste is considered highly proprietary by most manufacturers. However, discussion with a group at the University of Arizona Medical Center, who have been doing systematic comparisons of electrode jellies for use with defibrillators, led to the selection of $\mathrm{Cor}-\mathrm{Gel}^{\circledR}$ (Burdick). 
Use of this material eliminated the severe fluctuations in threshold and impedance we had encountered.

On dry skin, we typically measure impedances of $20-40 \mathrm{k} \Omega$ using $1-\mathrm{mm}^{2}$ electrodes. Using Cor-Gel ${ }^{\circledR}$, mean impedances generally average less than $10 \mathrm{k} \Omega$ with thresholds of $1-3 \mathrm{~mA}$. This means a $45 . \mathrm{V}$ battery could be used to power a portable system, simultaneously eliminating problems of skin breakdown, which may be experienced with higher voltages.

(Manuscript received January 24, 1982;

revision accepted for publication September 24, 1982.) 